\title{
Endovascular Aneurysm Repair Compared With Open Repair Does Not Improve Survival in Octogenarians
}

\author{
Hiroshi Banno, MD, PhD; Masayuki Sugimoto, MD, PhD; Tomohiro Sato, MD; \\ Shuta Ikeda, MD; Yohei Kawai, MD, PhD; Takuya Tsuruoka, MD; \\ Akio Kodama, MD, PhD; Kimihiro Komori, MD, PhD
}

\begin{abstract}
Background: Not every elderly person is frail, and whether it would be beneficial to perform endovascular aneurysm repair (EVAR) solely because a patient is older is unclear. This study aimed to compare the results of EVAR and open surgical repair (OSR) in elderly individuals.
\end{abstract}

\begin{abstract}
Methods and Results: From May 1998 to March 2021, 828 EVAR patients and 886 OSR patients with abdominal aortic aneurysm (AAA) were reviewed. Patients aged $\geq 80$ years were included among them. After propensity score matching by age, sex, and American Society of Anesthesiologists (ASA) classification, the outcomes were compared between patients who underwent EVAR and OSR. The study cohort was composed of 351 EVAR patients and 90 OSR patients. The groups had similar comorbidities, except that EVAR patients were significantly older and had higher ASA classifications. After propensity score matching, 79 pairs of patients were selected. The 30-day mortality ( 0 vs. 1.2\%) and aneurysm-related death (ARD) rates during follow up (2.3\% vs. $2.3 \%$, respectively) were similar between the groups. Kaplan-Meier curves revealed that estimated overall survival and freedom from ARD were also similar.
\end{abstract}

Conclusions: This study suggests that EVAR cannot improve survival outcomes compared with OSR if applied solely because a patient is aged $\geq 80$ years. Not only age but also other risk factors and quality of life after surgery need to be further studied.

Key Words: Abdominal aortic aneurysm; Endovascular aneurysm repair; Octogenarians; Open repair

$\mathbf{E}$ ndovascular aneurysm repair (EVAR) is widely accepted as a less invasive surgery for abdominal aortic aneurysm (AAA) and has significantly improved short-term outcomes for patients compared with open surgical repair (OSR). ${ }^{1-3}$ Although problems with its long-term performance have been recently reported, ${ }^{46}$ in general, they should not be a major concern for elderly people who do not have a long life expectancy. In contrast, better early OSR results have been observed with improvements in the operative procedure and perioperative management. Although EVAR should be more beneficial for higher-risk or more frail patients, not every elderly person is frail; thus, it is unclear whether it would be beneficial to perform EVAR solely because a patient is older. This study aimed to compare EVAR and OSR results for elderly individuals.

\section{Methods}

\section{Study Population}

Patients who underwent elective EVAR and OSR for AAA between May 1998 and March 2021 at our institution were reviewed. Patients aged $\geq 80$ years were included in this study. All patients provided written informed consent for their information to be recorded in a prospective collection database and met the inclusion criteria of the present study. The indication for repair was an AAA $\geq 5 \mathrm{~cm}$ in diameter, a rapidly growing aneurysm ( $\geq 5 \mathrm{~mm}$ per 6 months), and a saccular aneurysm. As EVAR became reimbursable in June 2007, our principle of AAA repair has been to use EVAR, if indicated, for patients aged $>75$ years or patients at high risk if OSR is performed, even if they are aged $<75$ years. ${ }^{7}$ In patients whose aneurysms cannot be repaired with standard EVAR (i.e., proximal neck length $<10 \mathrm{~mm}$ or severe conical neck shape), we prefer using OSR, even in patients aged $>75$ years. After careful examination, if it were decided that a patient could not tolerate OSR, complex EVAR using physician-modified fenestration or the chimney technique was applied. Those who required procedures for renal arteries during EVAR were excluded from this study. In the OSR group, patients requiring both infra- and supra-renal clamps were included (because

Received June 30, 2021; revised manuscript received September 9, 2021; accepted September 18, 2021; J-STAGE Advance Publication released online October 21, 2021 Time for primary review: 46 days

Division of Vascular and Endovascular Surgery, Department of Surgery, Nagoya University Graduate School of Medicine, Nagoya, Japan

Mailing address: Hiroshi Banno, MD, PhD, Division of Vascular and Endovascular Surgery, Department of Surgery, Nagoya University Graduate School of Medicine, 65 Tsurumai-cho, Showa-ku, Nagoya 466-8550, Japan. E-mail: hbanno@med.nagoya-u.ac.jp

All rights are reserved to the Japanese Circulation Society. For permissions, please e-mail: cj@j-circ.or.jp

ISSN-1346-9843 


\begin{tabular}{|c|c|c|c|c|c|c|}
\hline & \multicolumn{3}{|c|}{ Original cohorts } & \multicolumn{3}{|c|}{ Matched cohorts } \\
\hline & OSR $(n=90)$ & EVAR $(n=351)$ & $P$ value & OSR $(n=79)$ & EVAR $(n=79)$ & $P$ value \\
\hline Age (years) & $82(81,83)$ & $83(81,85)$ & $<0.0001^{*}$ & $82(81,83)$ & $82(81,83)$ & 0.88 \\
\hline Female sex & $22(24.4)$ & $71(20.2)$ & 0.38 & $16(20.3)$ & $16(20.3)$ & 1.0 \\
\hline Aneurysm diameter (mm) & $55(50,63)$ & $51(49,56)$ & $0.0026^{*}$ & $55(50,63)$ & $51(49,60)$ & $0.0036^{*}$ \\
\hline \multirow[t]{4}{*}{ ASA classification } & $1: 6(6.7)$ & $1: 60(17.1)$ & $0.033^{*}$ & $1: 6(7.6)$ & $1: 8(10.1)$ & 0.93 \\
\hline & $2: 49(54.4)$ & 2:189 (53.9) & & $2: 43(54.4)$ & $2: 43(54.4)$ & \\
\hline & 3:34 (37.8) & 3:99 (28.2) & & $3: 29(36.7)$ & 3:27 (34.2) & \\
\hline & $4: 1(1.1)$ & $4: 3(0.85)$ & & $4: 1(1.3)$ & $4: 1(1.3)$ & \\
\hline Smoker & $10(11.1)$ & $45(12.8)$ & 0.66 & $9(11.4)$ & $12(15.2)$ & 0.64 \\
\hline Hypertension & $71(78.9)$ & $260(74.1)$ & 0.35 & $63(79.8)$ & $62(78.5)$ & 1.0 \\
\hline Dyslipidemia & $37(41.1)$ & $154(43.9)$ & 0.64 & $33(41.8)$ & $37(46.8)$ & 0.63 \\
\hline Diabetes) & $12(13.3)$ & $32(9.1)$ & 0.23 & $12(115.2)$ & $7(8.9)$ & 0.33 \\
\hline CAD & 30 (33.3) & $113(32.2)$ & 0.84 & $26(32.9)$ & $24(30.4)$ & 0.86 \\
\hline CVD & $13(14.4)$ & $55(15.7)$ & 0.77 & $11(13.9)$ & $13(16.5)$ & 0.83 \\
\hline$C K D \geq 3 a$ & $55(61.1)$ & $218(62.1)$ & 0.86 & $47(59.5)$ & $46(58.2)$ & 1.0 \\
\hline Dialysis & $2(2.2)$ & $9(2.6)$ & 1.0 & $2(2.5)$ & $2(2.5)$ & 1.0 \\
\hline LVEF $<40 \%$ & $2(2.2)$ & $8(2.3)$ & 1.0 & $1(1.3)$ & $0(0)$ & 1.0 \\
\hline COPD & $39(43.3)$ & $170(48.4)$ & 0.39 & $36(45.6)$ & $41(51.9)$ & 0.52 \\
\hline HOT & 0 & $2(0.6)$ & 1.0 & 0 & $1(1.3)$ & 1.0 \\
\hline Serum albumin, $\mathrm{g} / \mathrm{dL}$ & $3.9(3.7,4.2)$ & $3.8(3.5,4.1)$ & 0.13 & $3.9(3.7,4.2)$ & $3.9(3.7,4.1)$ & 0.58 \\
\hline Total cholesterol, $\mathrm{mg} / \mathrm{dL}$ & $182(161,210)$ & $171(152,197)$ & $0.015^{*}$ & $182(162,209)$ & $174(160,196)$ & 0.25 \\
\hline Lymphocytes, count $/ \mu \mathrm{L}$ & $\begin{array}{c}1,300 \\
(1,100,1,700)\end{array}$ & $\begin{array}{c}1,300 \\
(1,000,1,600)\end{array}$ & 0.078 & $\begin{array}{c}1,300 \\
(1,100,1,700)\end{array}$ & $\begin{array}{c}1,300 \\
(1,000,1,900)\end{array}$ & 0.81 \\
\hline CONUT score & $2(1,3)$ & $2(1,3)$ & $0.008^{*}$ & $2(1,3)$ & $2(1,2)$ & 0.94 \\
\hline CONUT grade & & & $0.015^{\star}$ & & & 0.60 \\
\hline Normal & $38(45.8)$ & $119(34.0)$ & & $35(44.3)$ & $32(40.5)$ & \\
\hline Low risk & $40(48.2)$ & $190(54.3)$ & & $40(50.6)$ & $45(57.0)$ & \\
\hline Moderate risk & $5(6.0)$ & $40(11.4)$ & & $4(5.1)$ & $2(2.5)$ & \\
\hline Severe risk & $0(0)$ & $1(0.3)$ & & $0(0)$ & $0(0)$ & \\
\hline
\end{tabular}

$\mathrm{CAD}$, coronary artery disease; CKD, chronic kidney disease; CONUT, controlling nutritional status; COPD, chronic obstructive pulmonary disease; CVD, cerebrovascular disease; HOT, home oxygen therapy; LVEF, left ventricular ejection fraction. Data are presented as the median (interquartile range (IQR)) or number (\%). ${ }^{*} \mathrm{P}$ values represent statistical significance $(\mathrm{P}<0.05)$. CKD $\geq 3 a$ indicates estimated glomerular filtration rate $<60 \mathrm{~mL} / \mathrm{min} / 1.73 \mathrm{~m}^{2}$.

patients with a short $[10-15 \mathrm{~mm}]$ proximal neck can be treated with standard EVAR but require a suprarenal clamp in the OSR). The patients' demographic data and characteristics, operative data, and perioperative outcomes were extracted from their medical records. Perioperative complications were divided into 6 groups, including cardiac, pulmonary, renal, gastrointestinal, vascular, and other complications. Regarding long-term outcomes, overall survival and aneurysmrelated survival were evaluated. Aneurysm-related mortality included all deaths attributable to the aneurysm and aneurysm-related procedures, including the index surgery. Patient survival was also extracted from the medical record.

\section{Definitions}

Preoperative coronary artery disease (CAD) was defined as an abnormal result on coronary angiography and a history of myocardial infarction or open or percutaneous coronary artery revascularization. Chronic pulmonary disease was identified by pulmonary function studies or active treatment with medication. Hypertension and dyslipidemia were identified in patients undergoing active medical treatment or diet modification. Diabetes was defined as meeting at least 1 of the following criteria: (1) fasting glucose $\geq 126 \mathrm{mg} / \mathrm{dL}$; (2) plasma glucose $\geq 200 \mathrm{mg} / \mathrm{dL}$; (3) glycated hemoglobin $\geq 6.5 \%$; or (4) active treatment. Cerebrovascular disease was defined as a history of stroke, transient ischemic attack, carotid intervention, or significant disease detected by preoperative tests. The controlling nutritional status (CONUT) score is a tool for nutritional assessment that has been validated in a previous study and is reported to be useful in a variety of diseases. ${ }^{8}$ We calculated each patient's CONUT score by adding the albumin, lymphocyte, and cholesterol scores. Then, patients were classified into 4 groups based on the sum (the CONUT score) of each score. Patients with CONUT scores of 0 to 1 have a normal nutrition status, whereas those with a score of 2 to 4 are at mild risk, those with scores of 5 to 8 are at moderate risk, and those with scores of 9 to 12 are at severe risk of malnutrition.

\section{Data Analysis}

All statistical analyses were performed using JMP pro statistical software, version 15 (SAS Institute Inc., Cary, NC, USA). For comparisons, categorical variables were analyzed using the chi-squared test or Fisher's exact test, as appropriate. Continuous variables were analyzed using the Student's t-test or the Mann-Whitney U-test, as appropriate.

Patients undergoing EVAR were matched with patients 


\begin{tabular}{|c|c|c|c|c|c|c|}
\hline & \multicolumn{3}{|c|}{ Original cohorts } & \multicolumn{3}{|c|}{ Matched cohorts } \\
\hline & OSR $(n=90)$ & EVAR $(n=351)$ & $P$ value & OSR $(n=79)$ & EVAR $(n=79)$ & $P$ value \\
\hline Surgical duration (min) & $256(216,319)$ & $146(120,185)$ & $<0.0001^{*}$ & $256(217,322)$ & $138(116,173)$ & $<0.0001^{*}$ \\
\hline Blood loss (mL) & $1,833(1,181,2,792)$ & $109(41,227)$ & $<0.0001^{*}$ & $1,829(1,185,2,861)$ & $143(49,270)$ & $<0.0001^{*}$ \\
\hline $\begin{array}{l}\text { Inter-/Supra-renal clamp } \\
\text { Complications }\end{array}$ & $26(28.9)$ & & & $25(31.7)$ & & \\
\hline Any & $51(56.7)$ & $52(14.8)$ & $<0.0001^{*}$ & $48(60.8)$ & $13(16.5)$ & $<0.0001^{*}$ \\
\hline Cardiac & $5(5.6)$ & $10(2.8)$ & 0.20 & $3(3.8)$ & $2(2.5)$ & 1.0 \\
\hline Pulmonary & $3(3.3)$ & $4(1.1)$ & 0.15 & $3(3.8)$ & $2(2.5)$ & 1.0 \\
\hline Renal & $5(5.6)$ & $9(2.6)$ & 0.17 & $5(6.3)$ & $1(1.3)$ & 0.21 \\
\hline Gastrointestinal & $15(16.7)$ & $1(0.3)$ & $<0.0001^{*}$ & $14(17.7)$ & $0(0)$ & $<0.0001^{*}$ \\
\hline Vascular & $3(3.3)$ & $18(5.1)$ & 0.59 & $3(3.8)$ & $4(5.1)$ & 1.0 \\
\hline Delirium & $26(28.9)$ & $9(2.6)$ & $<0.0001^{*}$ & $26(32.9)$ & $2(2.3)$ & $<0.0001^{*}$ \\
\hline Others & $1(1.1)$ & $10(2.9)$ & 0.47 & $1(1.3)$ & $4(5.1)$ & 0.37 \\
\hline 30-day mortality & $1(1.1)$ & $0(0)$ & 0.20 & $1(1.3)$ & $0(0)$ & 1.0 \\
\hline
\end{tabular}

Data are presented as median (interquartile range (IQR)) or number (\%). ${ }^{*} \mathrm{P}$ values represent statistical significance $(P<0.05)$.

undergoing OSR according to their propensity scores, which were estimated using the following covariates: age, sex, American Society of Anesthesiologists (ASA) classification, and CONUT score. After matching, the outcomes were compared between patients who underwent EVAR and OSR. Kaplan-Meier analysis was applied to analyze survival and other endpoints. All $\mathrm{P}$ values were 2-sided, with a $\mathrm{P}$ value of $<0.05$ regarded as indicative of a significant difference.

\section{Results}

\section{Patient Characteristics and Demographics}

A total of 828 patients who underwent EVAR and 886 patients who underwent OSR for AAAs were identified during the study period. Of these, 351 patients $(79.6 \%)$ aged $\geq 80$ years who underwent EVAR and 90 patients $(20.4 \%)$ aged ${ }^{3} 80$ years who underwent OSR were included in this study. Due to our treatment strategy after the introduction of EVAR, 35 of $90(38.9 \%)$ cases treated with OSR were cases before the introduction of EVAR. The median age of the EVAR group (83 years, interquartile range [IQR]: $81-85$ years) was significantly higher than that of the OSR group ( 82 years, IQR: $81-83$ years) $(\mathrm{P}=0.0001)$. The median aneurysm size at the time of repair was $55 \mathrm{~mm}$ (IQR: $50-63 \mathrm{~mm}$ ) in the OSR group and $51 \mathrm{~mm}$ (IQR: $49-56 \mathrm{~mm})$ in the EVAR group $(\mathrm{P}=0.0026)$. The preoperative ASA classification was significantly different between the EVAR group and the OSR group. In addition, preoperative nutritional status was significantly worse in the EVAR group than in the OSR group. The 2 treatment groups were otherwise similar with respect to baseline comorbidities (Table 1).

The demographics of the patients matched with propensity scores are also shown in Table 1. Seventy-nine patients in each group were selected after propensity score matching (median age, 82 (IQR: $81-83$ ) years; 126 males (79.7\%)). In this matched cohort, median age, sex distribution, preoperative ASA classifications, and CONUT score were similar in both the EVAR group and OSR group. The median aneurysm size, however, was still larger in the OSR group (55 mm, IQR: $50-63 \mathrm{~mm}$ ) than in the EVAR group $(51 \mathrm{~mm}$, IQR: 49-60 mm).

\section{Operative Data}

The operative duration was significantly longer, and the amount of blood loss during surgery was significantly larger in the OSR group than in the EVAR group (in both the original and matched cohorts). The OSR group included 26 patients $(28.9 \%)$ who required inter- or supra-renal clamps in the original cohort and 25 patients $(31.7 \%)$ in the matched cohort (Table 2). Thirty-five patients $(10.0 \%)$ with a short proximal neck $<15 \mathrm{~mm}$ in length were included in the original EVAR group, and 13 of these patients (16.5\%) were included in the matched cohort.

\section{Clinical Outcomes}

Early Outcomes In the original cohorts, 30-day mortality was $1(1.1 \%)$ in the OSR group and 0 in the EVAR group $(\mathrm{P}=0.20)$. The cause of death of the patient who died after OSR was mesenteric ischemia. There was no other in-hospital death. The incidence of any perioperative complications was higher in the OSR group $(56.7 \%)$ than in the EVAR group $(14.8 \%)(\mathrm{P}<0.0001)$. Gastrointestinal complications and delirium accounted for the majority of the perioperative complications in the OSR group; however, the most frequent complication after EVAR was a vascular-associated complication (3 limb stenoses or occlusions, 4 access-associated complications, 5 hemorrhages requiring surgical repairs or blood transfusions, 3 strokes, 1 retrograde aortic dissection, and 1 primary type IIIa endoleak requiring re-intervention during the initial hospitalization). In the matched cohorts, 30-day mortalities were similar between both groups $(\mathrm{P}=1.0)$. The incidence rates of any perioperative complications were higher in the OSR group $(60.8 \%)$ than in the EVAR group $(16.5 \%)(\mathrm{P}<0.0001)$ (Table 2).

Late Outcomes The median duration of follow up in the original cohorts was 3.0 years (IQR: 1.0-5.0 years). Twenty-two patients $(24.4 \%)$ in the OSR group and 78 $(22.2 \%)$ in the EVAR group were lost to follow up at 3 years after surgery. There were $2(2.2 \%)$ aneurysm-related deaths (ARDs) during follow up in the OSR group and 10 $(2.9 \%)$ in the EVAR group $(\mathrm{P}=1.0)$. Among the 35 patients with the proximal short neck $(<15 \mathrm{~mm})$ in the EVAR group, 18 patients were outside the instruction for use by the manufacturer ( 4 cases $<10 \mathrm{~mm}$ and 14 cases $\geq 10 \mathrm{~mm}$ and 

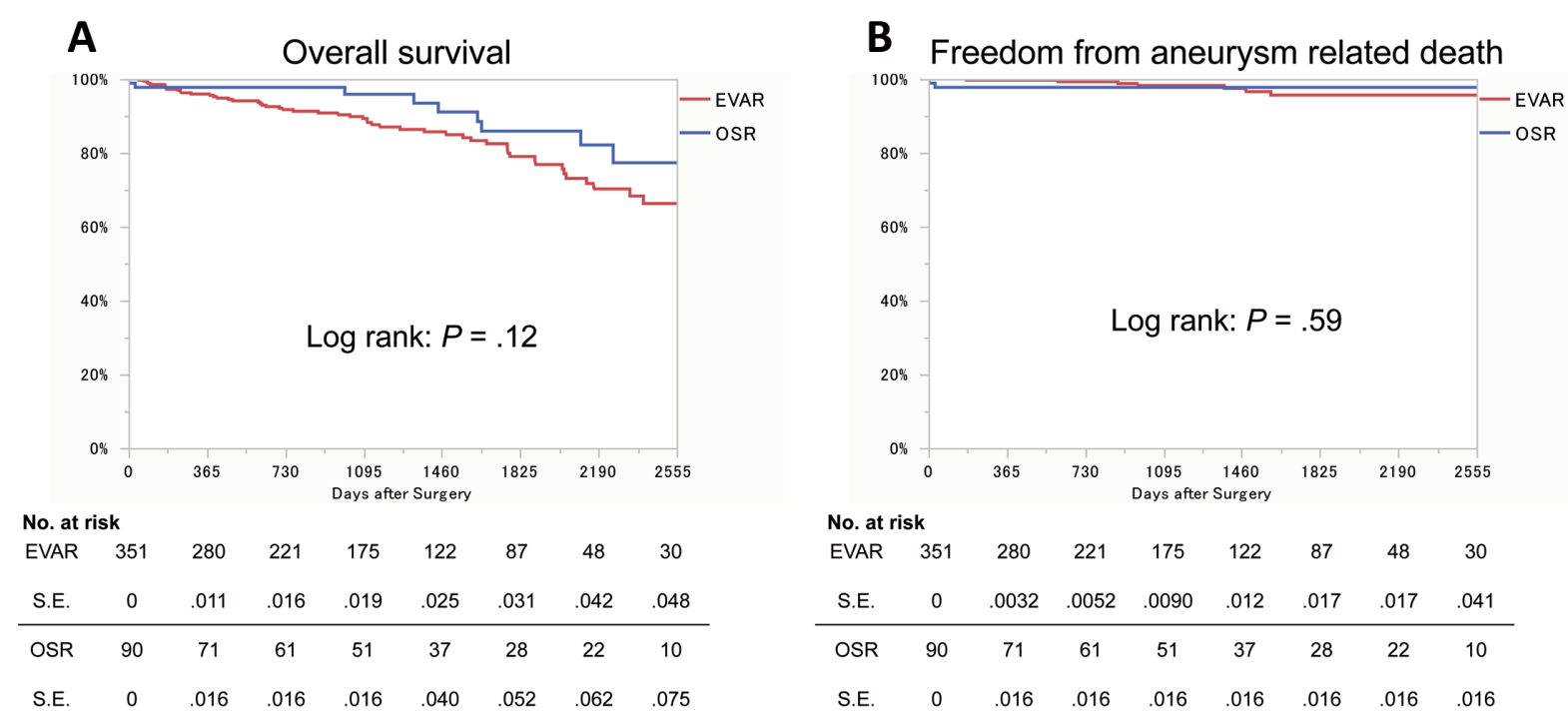

Figure 1. Kaplan-Meier survival curves of octogenarians who underwent endovascular aneurysm repair (EVAR) (red) and open surgical repair (OSR) (blue) in the original cohorts. Color bands indicate the 95\% confidence interval for each group. (A) Overall survival and (B) freedom from aneurysm-related. S.E., standard error.
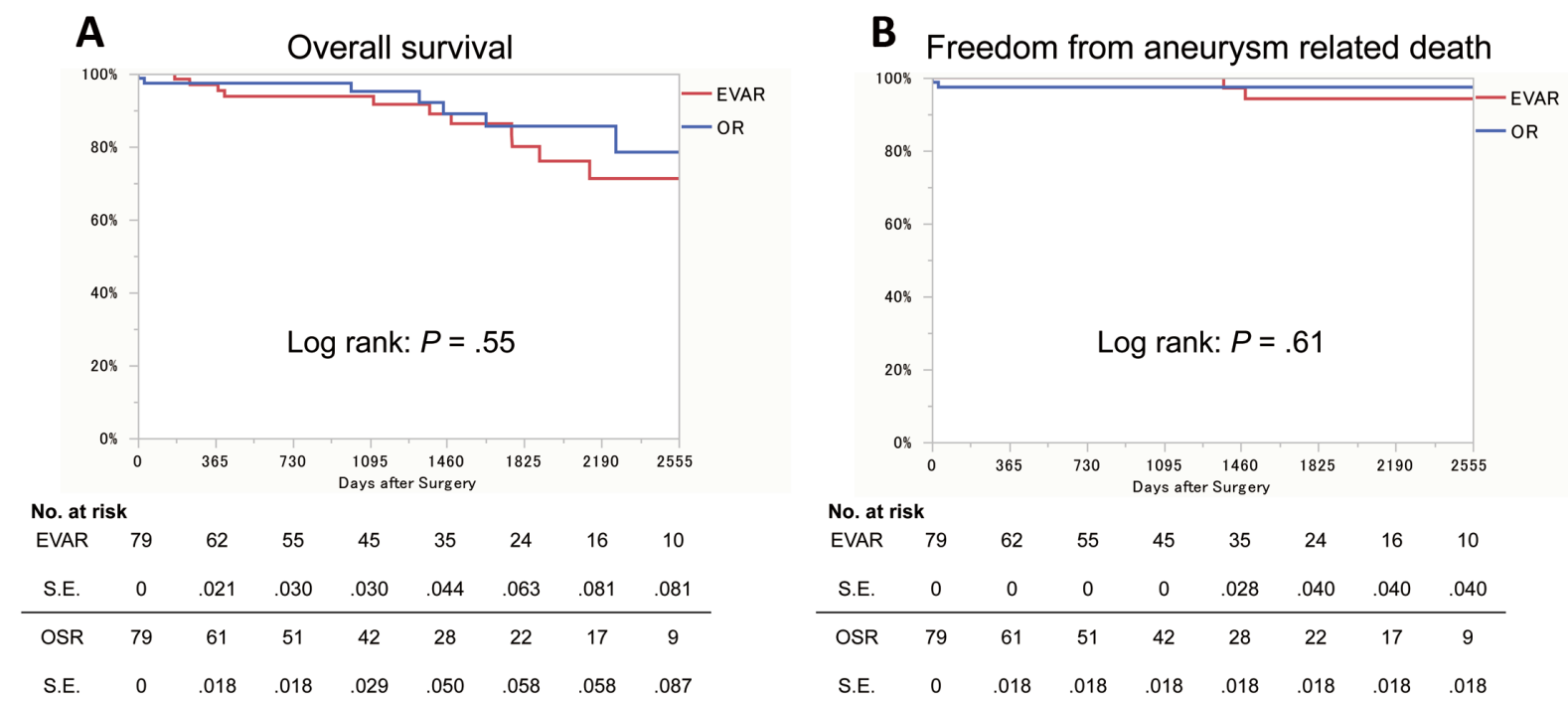

Figure 2. Kaplan-Meier survival curves of octogenarians who underwent endovascular aneurysm repair (EVAR) (red) and open surgical repair (OSR) (blue) in the matched cohorts. Color bands indicate the 95\% confidence interval for each group. (A) Overall survival and (B) freedom from aneurysm-related. S.E., standard error.

$<15 \mathrm{~mm}$ were treated with devices other than Endurant $\left.{ }^{\circledR}\right)$. However, there was no ARD for those patients. KaplanMeier curves revealed that estimated overall survival (log rank: $\mathrm{P}=0.12)$ and freedom from ARD (log rank: $\mathrm{P}=0.59)$ were similar between the 2 groups (Figure 1). After matching, the median duration of follow up was 3.1 years (IQR: $1.0-5.4$ years). There were 21 patients $(26.6 \%)$ in the OSR group and 16 patients $(20.3 \%)$ in the EVAR group who were lost to follow up at 3 years after surgery. In the matched cohorts, there were $2(2.5 \%)$ ARDs during follow up in the
OSR group, and $3(3.8 \%)$ ARDs in the EVAR group $(\mathrm{P}=1.0)$. Kaplan-Meier curves revealed that estimated overall survival (log rank: $\mathrm{P}=0.55)$ and freedom from ARD (log rank: $P=0.61)$ were also similar between the 2 groups (Figure 2).

\section{Discussion}

In this single-institution retrospective study, EVAR did not show advantages in terms of either short- or long-term 
survival for patients aged $\geq 80$ years compared with OSR. After propensity matching by age, sex, and preoperative ASA classification, the 30-day mortality and ARD during follow up were similar between the 2 groups. Estimated overall survival and freedom from ARD were also similar between the 2 groups. One of the reasons for this result is that unlike the reports thus far, the number of perioperative deaths after OSR was very low.,10 It is not clear what caused the difference. It may depend on ethnicity or the medical system. In addition, this might be related to ARDs occurring in the late period after EVAR. Although poorer outcomes after AAA repair are generally reported in elderly people, the underlying cause is unknown. ${ }^{11}$ Comorbidities naturally increase with age, which may be one of the reasons for poor outcomes. Otherwise, it may be because the number of patients with hostile anatomies, tortuous vessels and a higher degree of atherosclerotic disease increases with age. In fact, approximately $30 \%$ of patients in the OSR group required inter- or supra-renal clamping. Ten percent of patients had a short proximal neck even in the EVAR group. Despite this complex morphology, perioperative mortality was $1.1 \%$ for OSR and 0 for EVAR, which is very low. In our experience thus far, the causes of death among patients undergoing OSR for AAA are usually mesenteric ischemia or multiple organ failure caused by shower embolism. The cause of the 1 death in this study was mesenteric ischemia. Even if EVAR was selected in this case, it would have been difficult to prevent this complication. In addition, Hoshina et al reported that EVAR patients with massive neck atheroma tend to develop embolisms in the late period and that care should be taken after discharge. ${ }^{12}$

As mentioned above, the selection criteria for EVAR and OSR in our institution prioritizes EVAR for patients aged $\geq 75$ years. When an additional procedure to the renal artery is necessary due to a hostile neck, OSR is performed whenever possible, but complicated EVAR is considered for high-risk patients who may have difficulty tolerating OSR. As a result, there were obviously many cases of renal artery clamping in the OSR group in this study. According to the results in this study, however, our policy of EVAR priority solely based on age may need to be reconsidered. It would be best to have an established frailty score that could predict a patient's prognosis, but unfortunately, there is currently no right way to convince everyone. A nutritional assessment such as the CONUT score used in this study may help to predict the patient's life expectancy. In this cohort, the moderate risk group tended to have a poor prognosis, but no statistically significant difference was observed (Supplementary Figure). Numerous reports have demonstrated that EVAR causes late-occurring complications, ${ }^{4,5,13}$ and EVAR selection criteria may need to be revised in institutions with excellent perioperative outcomes after OSR, such as ours. In addition, the population of our country is rapidly aging, and the average life expectancy of octogenarians has continued to increase. Hence, the percentage of elderly individuals is among the highest in the world. The life expectancy of people at 80 years is approximately 9 years for men and 12 years for women in Japan. Rueda-Ochoa et al reported that survival after uncomplicated EVAR in octogenarians is similar to the general populations of octogenarians without AAA, ${ }^{14}$ and it is important to further examine the factors related to late ARDs after EVAR. Studies to identify risk factors for endoleaks that result in an increased aneurysm diameter, rather than just risk factors for type II endoleaks, may also be important. ${ }^{15}$

In contrast, OSR causes perioperative complications significantly more often than EVAR. Because delirium, a complication that is essentially reversible and not lifethreatening, is also included as a complication in this study, it makes a considerable difference. Although fortunately not lethal, gastrointestinal complications, which are potentially life-threatening, were significantly more common in the OSR group. These complications might worsen the quality of life after discharge.

From the results of this study, it is concluded that the procedure selection for AAA repair in the elderly population should include the evaluation of risk factors for poor late outcomes after EVAR, patient prognosis (not only estimated for age) and postoperative quality of life derived from patient fragility. There is a considerable advantage in choosing EVAR, which is less invasive and has fewer complications, even for elderly people who are healthy and expected to have a long-life expectancy if their morphology is conducive to achieving satisfactory long-term results after EVAR. In patients with morphological risk factors that might be related to poor outcomes after EVAR, the advantages of choosing OSR may outweigh the disadvantages, even for older patients. In contrast, it is not considered problematic to select EVAR, which has problems in the late period, for patients who do not have a long-life expectancy due to their frailty. Kodama et al reported associations of nutritional status and muscle size with mortality after OSR. They demonstrated that controlling for the nutritional status score and skeletal muscle size were useful in identifying and optimizing the treatment of highrisk patients who will not benefit from OSR so that EVAR or no intervention can be recommended. ${ }^{16}$

The present study has several limitations. First, our study was a non-randomized, retrospective observational study at a single institution. Clearly, there are numerous selection biases in the indication for the choice of procedure for AAA repair. Second, because recent cases are included in this study, some cases have only had a short follow-up time. In addition, not a few patients were lost to follow up. The percentage of quite a few patients, however, was similar between the 2 groups. Finally, to minimize the influence of factors that might impact survival, patients' backgrounds were adjusted using propensity score matching, followed by a comparison of the overall and aneurysmrelated survival rates. However, this matching excluded older patients (i.e., those aged in their late $80 \mathrm{~s}$ ), who were more abundant in the EVAR group in the analysis.

\section{Conclusions}

This study demonstrates that EVAR cannot improve survival outcomes compared with OSR if this less invasive procedure were applied solely because a patient is aged $\geq 80$ years. Age and other risk factors predict survival after EVAR, and quality of life after surgery needs to be further studied.

\section{Disclosures}

K.K. is a member of Circulation Journal's Editorial Team. All other authors have no conflicts of interest to declare.

\section{IRB Information}

The institutional review board of Nagoya University approved this 
study (Reference number: 2021-0154).

\section{References}

1. Becquemin JP, Pillet JC, Lescalie F, Sapoval M, Goueffic Y, Lermusiaux $\mathrm{P}$, et al. A randomized controlled trial of endovascular aneurysm repair versus open surgery for abdominal aortic aneurysms in low- to moderate-risk patients. J Vasc Surg 2011; 53: $1167-1173 . e 1$

2. Blankensteijn JD, de Jong SECA, Prinssen M, van der Ham AC, Buth J, van Sterkenburg SMM, et al. Two-year outcomes after conventional or endovascular repair of abdominal aortic aneurysms. N Engl J Med 2005; 352: 2398-2405.

3. Greenhalgh RM, Brown LC, Kwong GPS, Powell JT, Thompson SG, EVAR trial participants. Comparison of endovascular aneurysm repair with open repair in patients with abdominal aortic aneurysm (EVAR trial 1), 30-day operative mortality results: Randomised controlled trial. Lancet 2004; 364: 843-848.

4. Patel R, Sweeting MJ, Powell JT, Greenhalgh RM. Endovascular versus open repair of abdominal aortic aneurysm in 15-years' follow-up of the UK endovascular aneurysm repair trial 1 (EVAR trial 1): A randomised controlled trial. Lancet 2016; 388: 2366 2374.

5. Lederle FA, Kyriakides TC, Stroupe KT, Freischlag JA, Padberg FT, Matsumura JS, et al. Open versus endovascular repair of abdominal aortic aneurysm. N Engl J Med 2019; 380: 2126-2135.

6. Schermerhorn ML, Buck DB, James O'Malley A, Curran T, McCallum JC, Darling J, et al. Long-term outcomes of abdominal aortic aneurysm in the medicare population. $N$ Engl J Med 2015; 373: $328-338$.

7. Yamamoto K, Komori K, Banno H, Narita H, Kodama A, Sugimoto M. Validation of patient selection for endovascular aneurysm repair or open repair of abdominal aortic aneurysm: Single-center study. Circ J 2015; 79: 1699-1705.

8. Ignacio de Ulíbarri J, Gonzalez-Madroño A, de Villar NGP, González P, González B, Mancha A, et al. CONUT : A tool for controlling nutritional status. First validation. Nutr Hosp 2005; 20: $38-45$.
9. Hicks CW, Obeid T, Arhuidese I, Qazi U, Malas MB. Abdominal aortic aneurysm repair in octogenarians is associated with higher mortality compared with nonoctogenarians. J Vasc Surg 2016; 64: 956-965.e1.

10. Scallan O, Novick T, Power AH, DeRose G, Duncan A, Dubois L. Long-term outcomes comparing endovascular and open abdominal aortic aneurysm repair in octogenarians. J Vasc Surg 2020; 71: $1162-1168$

11. Henebiens M, Vahl A, Koelemay MJW. Elective surgery of abdominal aortic aneurysms in octogenarians: A systematic review. $J$ Vasc Surg 2008; 47: 676-681.

12. Hoshina K, Hosaka A, Takayama T, Kato M, Ohkubo N, Okamoto $\mathrm{H}$, et al. Outcomes after open surgery and endovascular aneurysm repair for abdominal aortic aneurysm in patients with massive neck atheroma. Eur J Vasc Endovasc Surg 2012; 43: $257-261$.

13. Hoshina K, Ishimaru S, Sasabuchi Y, Yasunaga H, Komori K Outcomes of endovascular repair for abdominal aortic aneurysms. Ann Surg 2019; 269: 564-573.

14. Rueda-Ochoa OL, van Bakel P, Hoeks SE, Verhagen H, Deckers J, Rizopoulos D, et al. Survival after uncomplicated EVAR in cctogenarians is similar to the general population of octogenarians without an abdominal aortic aneurysm. Eur J Vasc Endovasc Surg 2020; 59: 740-747.

15. Fujii T, Banno H, Kodama A, Sugimoto M, Akita N, Tsuruoka $\mathrm{T}$, et al. Aneurysm sac thrombus volume predicts aneurysm expansion with type II endoleak after endovascular aneurysm repair. Ann Vasc Surg 2020; 66: 85-94.e1.

16. Kodama A, Takahashi N, Sugimoto M, Niimi K, Banno H, Komori K. Associations of nutritional status and muscle size with mortality after open aortic aneurysm repair. J Vasc Surg 2019; 70: $1585-1593$.

\section{Supplementary Files}

Please find supplementary file(s);

http://dx.doi.org/10.1253/circj.CJ-21-0574 